\title{
Evaluation of morphological characteristics of septal rims affecting successful transcatheter atrial septal defect closure in children and adults
}

\author{
Mehmet Burhan Oflaz', Feyza Aysenur Pac², Ayse Esin Kibar, Sevket Balli4, Ibrahim Ece ${ }^{5}$ \\ 1Department of Pediatric Cardiology, Cumhuriyet University, School of Medicine, Sivas, Turkey \\ 2Department of Pediatric Cardiology, Yuksek Ihtisas Education and Research Hospital, Ankara, Turkey \\ 3Department of Pediatric Cardiology, Mersin Children Hospital, Mersin, Turkey \\ ${ }^{4}$ Department of Pediatric Cardiology, Ataturk Hospital, Balikesir, Turkey \\ ${ }^{5}$ Department of Pediatric Cardiology, Yuzuncu Yil University, School of Medicine, Van, Turkey
}

Postep Kardiol Inter 2013; 9, 3 (33): 205-211

DOI: $10.5114 /$ pwki.2013.37497

\begin{abstract}
Introduction: Determining other echocardiographic predictors along with the measured atrial septal defect (ASD) size and evaluating the closure together with these predictors would increase the chance of success for transcatheter closure of ASD.

Aim: To evaluate echocardiographic parameters affecting defect closure in children and adult patients with secundum ASD.

Material and methods: In all patients, size of ASD, total length of atrial septum (TS), superior-posterior, inferior-posterior, superior-anterior and inferior-anterior rims surrounding the defect were measured by transesophageal echocardiography (TEE), and several measurement ratios were derived on the basis of TEE parameters.

Results: A total 216 patients with secundum ASD were included in this study. The device was successfully implanted in 65 children and 65 adults. Both in pediatric and adult cases, the ratio of successful closure was found to be significantly higher when the ratio of defect size to TS was $\leq 0.35$, the ratio of superior-anterior (SA) rim to the defect size was $>0.75$ and the ratio of inferior-posterior (IP) rim to the defect size was $>1.0$. It was found that having more than one of these predictors in a single case increased the chance of closure success significantly $(p<0.001)$.

Conclusions: We concluded that a ratio of the defect size to TS $\leq 0.35$, a ratio of SA rim to defect size $>0.75$ and a ratio of IP rim to defect size $>1.0$ were found to be echocardiographic predictors that could be used in successful transcatheter ASD closure both in children and adults.
\end{abstract}

Key words: atrial septal defect, transcatheter closure, echocardiographic predictors.

\section{Introduction}

Openings that allow shunting of blood between the left and right atrium, except patent foramen ovale, are called atrial septal defect (ASD) [1, 2]. Atrial septal defect comprises 6-10\% of all congenital heart abnormalities [2]. Treatment of ASD is either percutaneous or surgical closure of the defect. Accurate measurement of the defect size plays a key role in closing ASD using a percutaneous occluder device. It is possible to determine the size of the defect by transesophageal echocardiography (TEE), which is a noninvasive technique. In the literature, it has been emphasized that TEE is a gold standard in transcatheter closure of ASD and thus should be used in analyzing septal defect and rims dur- ing the process. Therefore, using echocardiographic parameters affecting success of closure may prevent possible complications in percutaneous closure of ASDs. In terms of success, there is no definite ASD size or predictor as the size of ASD differs from 1 patient to another. Determining other predictors along with the measured ASD size and evaluating the closure together with such predictors would increase the chance of success.

\section{Aim}

In the present study, demographic features, echocardiographic and angiographic measurements of cases with secundum ASD and those planned to undergo percutaneous

\section{Corresponding author:}

Mehmet Burhan Oflaz MD, Cumhuriyet University, School of Medicine, Cumhuriyet Universitesi Tip Fakultesi Hastanesi, Cocuk Kardiyoloji Poliklinigi, Kampus, 58140 Sivas, Turkey, tel.: +905055170783, e-mail: mburhanoflaz@gmail.com

Received: 12.04.2013, accepted: 9.05.2013. 
closure were studied, and it was aimed to evaluate echocardiographic parameters affecting defect closure.

\section{Material and methods}

\section{Study patients}

Pediatric and adult cases diagnosed with secundum ASD between December 2005 and January 2011 at the Ankara Yuksek Ihtisas Education and Research Hospital, Turkey and having indications for closure were included in the study. Age, body weight, height, complaints on admission, physical examination, electrocardiography and telecardiography findings, echocardiographic defect and rim measurements, angiographic stretched defect diameters, accompanying noncardiovascular system diseases and medical treatments were recorded. Criteria used in selecting patients appropriate for percutaneous closure were detection of secundum ASD with left-to-right shunt in echocardiography, having a defect $\geq 5 \mathrm{~mm}$ away from mitral, tricuspid valve, coronary sinus, right upper pulmonary vein, inferior vena cava and superior vena cava, and increased right ventricular volume (pulmonary/systemic blood flow ratio $>1.5$ and/or sign of right ventricular volume overload) [2]. Exclusion criteria were having a congenital heart disease other than ASD, sinus venosus and ostium primum type ASDs, severe pulmonary hypertension, systemic or local infections, bleeding disorder, untreated ulcer or any other contraindications to acetyl salicylic acid therapy and having demonstrated intracardiac thrombi on echocardiography.

\section{Study protocol}

Informed written consent to the procedure was obtained from all patients or parents, and all the patients were given prophylaxis for infective endocarditis before closure.

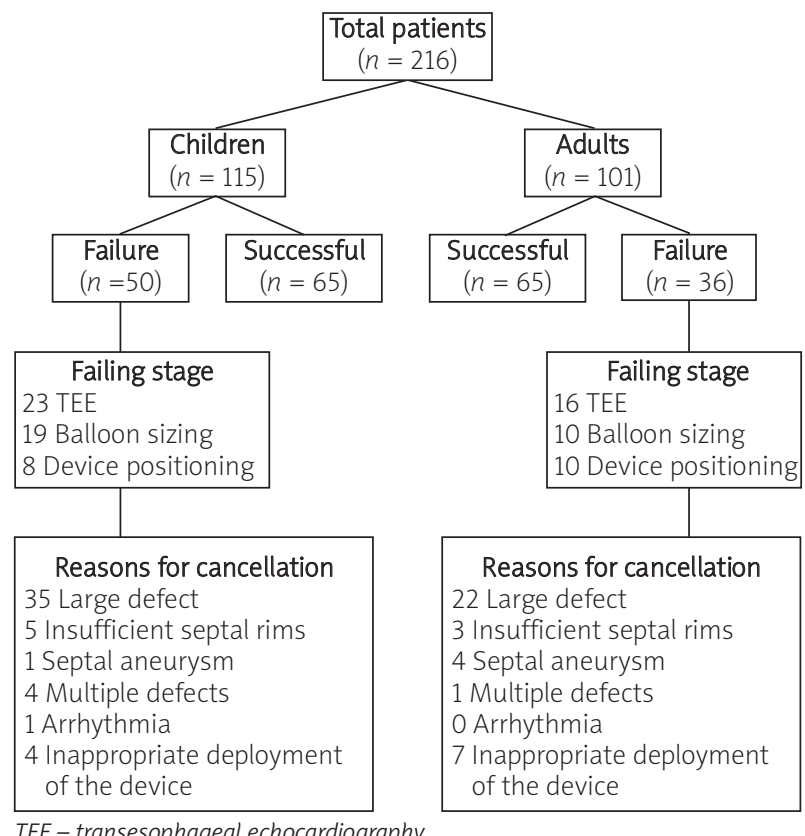

Fig. 1. Flowchart of patients
Echocardiographic evaluation was made using Philips Envsior C-HD device (Philips, Andover MA, USA) and 3.5-5.5$7.5 \mathrm{MHz}$ probes. All the patients were subjected to echocardiographic examination by two-dimensional, M-mode and continuous/pulsed wave Doppler and color flow Doppler. Before catheterization, heparin was given at $50 \mathrm{U} / \mathrm{kg}$ intravenously. Atrial, ventricular, aortic and pulmonary arterial pressures were measured, blood samples were taken and pulmonary/systemic flow (Qp/Qs) ratios were calculated.

\section{Two-dimensional transesophageal echocardiography}

Morphology of the interatrial septum was evaluated echocardiographically using longitudinal and horizontal sections. Longitudinal length was assessed in the caval right atrial and atrial septal views while horizontal sections were evaluated by TEE in four-chamber view. The total diameter of inter atrial septum and superior-posterior, inferiorposterior, superior-anterior and inferior-anterior rims surrounding the defect were measured by TEE in midesophageal $0^{\circ} 4$-chamber view position, midesophageal $30-50^{\circ}$ aortic position and $90-120^{\circ}$ bicaval position [3]. The largest defect diameter obtained in TEE measurements was selected as the reference diameter. The superior-anterior rim was measured in the horizontal plane as the distance between the aortic annulus and the defect. The inferior-anterior rim was measured in the four-chamber section as the distance between the defect and atrioventricular valves. The longitudinal (bicaval) views were used to determine the superior-posterior rim as the distance from the superior part of the defect to the superior vena cava and the inferior-posterior rim as the distance from the inferior part of the defect to the inferior vena cava. The posterior rim represented the distance from the defect to the coronary sinus and posterior atrial wall in the horizontal view. Coronary sinus rim was defined as the distance between the defect and the coronary sinus opening in the four-chamber view. Total length of the atrial septum (TS) was determined in the four-chamber and bicaval right atrial views. In order to find the real size of the defect and the strength of the rims, the stretched diameter of ASD was measured using a balloon catheter [3-5].

The Amplatzer septal occluder (ASO, AGA Medical, Golden Valley, Minnesota, USA) is one of the most frequently used devices to close ASD and has been proven to be highly effective and safe in the early-long term. The Figulla septal occluder (FSO, International Occlutech AB, Helsingborg, Sweden) device is another device designed to close the full range of defects. It looks similar to the ASO. There will be no learning curve using this device since the implantation technique is similar to the ASO. Cases found to be appropriate for closure underwent occluder device closure while surgical closure was performed in the other patients. Care was taken to ensure that the device did not compress the aorta or obstruct the right pulmonary veins, caval veins, coro- 
Table 1. Demographic and clinical features, and closure data of pediatric and adult patients

\begin{tabular}{|c|c|c|c|c|c|c|}
\hline Parameter & $\begin{array}{l}\text { Children } \\
(n=115)\end{array}$ & $\begin{array}{c}\text { Adults } \\
(n=101)\end{array}$ & $\begin{array}{l}\text { Value } \\
\text { of } p\end{array}$ & $\begin{array}{c}\text { Successful } \\
(n=130)\end{array}$ & $\begin{array}{l}\text { Failed } \\
(n=86)\end{array}$ & $\begin{array}{l}\text { Value } \\
\text { of } p\end{array}$ \\
\hline Age [years] & $10.1 \pm 4.0$ & $36.6 \pm 11.4$ & $<0.05$ & $24.4 \pm 16.3$ & $19.7 \pm 14.2$ & NS \\
\hline Gender (female/male) & $72 / 43$ & $52 / 49$ & NS & $78 / 52$ & $46 / 40$ & NS \\
\hline Weight $[\mathrm{kg}]$ & $33.4 \pm 15.4$ & $68.9 \pm 12.7$ & $<0.05$ & $52.8 \pm 22.4$ & $45.1 \pm 22.5$ & NS \\
\hline Height $[\mathrm{cm}]$ & $137.35 \pm 24.9$ & $163.31 \pm 8.4$ & $<0.05$ & $152.0 \pm 21.4$ & $146.5 \pm 24.8$ & NS \\
\hline Defect size, TEE $[\mathrm{cm}]$ & $1.35 \pm 0.6$ & $1.59 \pm 0.8$ & $<0.05$ & $1.17 \pm 0.4$ & $1.89 \pm 0.7$ & $<0.05$ \\
\hline Stretched defect size, TEE [cm] & $1.64 \pm 4.4$ & $2.12 \pm 5.1$ & $<0.05$ & $1.73 \pm 4.4$ & $2.38 \pm 4.9$ & $<0.05$ \\
\hline Stretched defect size, angiography $[\mathrm{cm}]$ & $1.68 \pm 4.5$ & $2.16 \pm 5.2$ & $<0.05$ & $1.75 \pm 4.5$ & $2.41 \pm 5.0$ & $<0.05$ \\
\hline Total septum diameter $[\mathrm{cm}]$ & $3.50 \pm 0.5$ & $4.54 \pm 0.8$ & $<0.05$ & $3.93 \pm 0.7$ & $4.03 \pm 0.9$ & NS \\
\hline Systolic PAB [mm Hg] & $30.1 \pm 7.6$ & $31.9 \pm 8.4$ & NS & $30.5 \pm 8.1$ & $31.6 \pm 7.8$ & NS \\
\hline Diastolic PAB [mm Hg] & $12.9 \pm 4.9$ & $14.4 \pm 5.6$ & NS & $14.2 \pm 5.1$ & $12.7 \pm 5.5$ & NS \\
\hline Mean PAB [mm Hg] & $20.4 \pm 5.8$ & $21.8 \pm 5.8$ & NS & $21.3 \pm 5.7$ & $20.7 \pm 6.0$ & NS \\
\hline Qp/Qs & $2.32 \pm 0.7$ & $2.11 \pm 0.7$ & NS & $1.93 \pm 0.4$ & $2.68 \pm 0.8$ & $<0.05$ \\
\hline Procedure time [min] & $65 \pm 10$ & $51 \pm 13$ & NS & $68 \pm 11$ & $49 \pm 8$ & $<0.05$ \\
\hline Fluoroscopy time [min] & $19 \pm 7.5$ & $14 \pm 5.0$ & NS & $22 \pm 4.3$ & $13 \pm 3.1$ & $<0.05$ \\
\hline Successful closure & $65(56.5 \%)$ & $65(64.3)$ & NS & & & \\
\hline \multirow[t]{2}{*}{ Device type } & ASO: $26(40 \%)$ & ASO: $16(25 \%)$ & NS & & & \\
\hline & FSO: $39(60 \%)$ & FSO: $49(75 \%)$ & & & & \\
\hline \multicolumn{7}{|l|}{ Failing stage } \\
\hline$\overline{T E E}$ & $23(20 \%)$ & $16(15.8 \%)$ & NS & & & \\
\hline Balloon sizing & 19 (16.5\%) & $10(9.9 \%)$ & NS & & & \\
\hline Device positioning & $8(6.9 \%)$ & $10(9.9 \%)$ & NS & & & \\
\hline \multicolumn{7}{|l|}{ Reasons for cancellation } \\
\hline Large defect & $35(30.4 \%)$ & $22(21.7 \%)$ & NS & & & \\
\hline Insufficient septal rims & $5(4.3 \%)$ & $3(2.9 \%)$ & NS & & & \\
\hline Septal aneurysm & $1(0.8 \%)$ & $4(3.9 \%)$ & $<0.05$ & & & \\
\hline Multiple defects & $4(3.4 \%)$ & $1(0.9 \%)$ & $<0.05$ & & & \\
\hline Arrhythmia & $1(0.8 \%)$ & $0(0 \%)$ & NS & & & \\
\hline Inappropriate deployment of the device & $4(3.4 \%)$ & $7(6.9 \%)$ & NS & & & \\
\hline Residual shunt at procedure & $4(3.4 \%)$ & $3(2.9 \%)$ & NS & & & \\
\hline Complete occlusion [\%] & 99.2 & 99 & NS & & & \\
\hline
\end{tabular}

Data are expressed as mean $\pm S D$ and frequencies (percentile). TEE - transesophageal echocardiography, PAB - pulmonary artery pressure, Qp/Qs - pulmonary-to-systemic flow ratio, FSO - Figulla Septal Occluder, ASO - Amplatzer Septal Occluder, NS - not significant

nary sinus, or the mitral valve. Any residual shunt was evaluated by two-dimensional color-flow imaging. Patients fitted with a device were given $5 \mathrm{mg} / \mathrm{kg} /$ day oral acetyl salicylic acid for a 6-month term and they were advised to avoid subacute bacterial endocarditis prophylaxis and competitive sports for the same period. During the follow-ups 1 day and 1, 3 and 6 months after the procedure, physical and electrocardiographic examinations were performed and cases having tachycardia and/or dysrhythmia underwent electrocardiographic monitoring for $24 \mathrm{~h}$ or longer [6].

\section{Statistical analysis}

Descriptive analysis was performed for demographic and clinical characteristics of the patients. Distribution of data was assessed by using a one-sample Kolmogorov-Smirnov test. Data are demonstrated as mean \pm SD for normally distributed continuous variables and frequencies (percentile) for categorical variables. Values between different groups were compared using the independent-samples t test. MannWhitney $U$ test was used if the data were not normally distributed. The $\chi^{2}$ test was used to assess differences between categorical variables. Multivariate logistic regression analysis was performed to identify factors predictive of successful ASD closure. The data were further compared by plotting scattergrams and by using median and quartile splits to develop predictive categories. A $p$-value $<0.05$ was considered to be significant. Analyses were perform-ed with the software package SPSS 11.0 (SPSS, Inc, Chicago, IL, USA). 
Table 2. Measurements of length of rims surrounding the defect, total septum length, ratio of superior-anterior rim to the defect, ratio of inferior-posterior rim to the defect, and ratio of atrial septal defect to total septum, in different echocardiographic windows and comparisons between the groups

\begin{tabular}{|c|c|c|c|c|c|c|}
\hline \multirow{2}{*}{$\begin{array}{l}\text { Parameter/echocardiographic } \\
\text { windows }\end{array}$} & \multicolumn{2}{|c|}{ Children } & \multirow{2}{*}{$\begin{array}{l}\text { Value } \\
\text { of } p\end{array}$} & \multicolumn{2}{|c|}{ Adults } & \multirow{2}{*}{$\begin{array}{l}\text { Value } \\
\text { of } p\end{array}$} \\
\hline & $\begin{array}{l}\text { Successful } \\
\text { closure }\end{array}$ & Failed & & $\begin{array}{c}\text { Successful } \\
\text { closure }\end{array}$ & Failed & \\
\hline \multicolumn{7}{|l|}{ Superior-anterior rim $[\mathrm{cm}]$} \\
\hline Four-chamber & $1.37 \pm 0.35$ & $0.92 \pm 0.35$ & $<0.05$ & $1.57 \pm 0.54$ & $1.45 \pm 0.72$ & NS \\
\hline Bicaval & $1.12 \pm 0.33$ & $0.89 \pm 0.42$ & $<0.05$ & $1.31 \pm 0.40$ & $1.26 \pm 0.41$ & NS \\
\hline Short-axis & $0.59 \pm 0.36$ & $0.46 \pm 0.22$ & NS & $0.76 \pm 0.59$ & $0.55 \pm 0.33$ & NS \\
\hline \multicolumn{7}{|l|}{ Inferior-posterior rim [cm] } \\
\hline Four-chamber & $1.24 \pm 0.42$ & $0.96 \pm 0.35$ & $<0.01$ & $1.50 \pm 0.56$ & $1.27 \pm 0.61$ & NS \\
\hline Bicaval & $1.22 \pm 0.41$ & $0.98 \pm 0.48$ & $<0.05$ & $1.41 \pm 0.50$ & $1.22 \pm 0.62$ & NS \\
\hline Short-axis & $1.66 \pm 0.29$ & $1.02 \pm 0.50$ & $<0.05$ & $0.76 \pm 0.59$ & $0.55 \pm 0.33$ & NS \\
\hline \multicolumn{7}{|l|}{ Total septal length $[\mathrm{cm}]$} \\
\hline Four-chamber & $3.45 \pm 0.54$ & $3.57 \pm 0.52$ & NS & $4.35 \pm 0.73$ & $4.60 \pm 1.01$ & NS \\
\hline Bicaval & $3.42 \pm 0.60$ & $3.48 \pm 0.61$ & NS & $4.11 \pm 0.63$ & $4.23 \pm 0.80$ & NS \\
\hline Short-axis & $2.98 \pm 0.41$ & $3.25 \pm 0.53$ & NS & $3.44 \pm 0.37$ & $3.85 \pm 1.26$ & NS \\
\hline \multicolumn{7}{|l|}{ Superior-anterior rim/defect size } \\
\hline Four chamber & $1.23 \pm 0.61$ & $0.63 \pm 0.40$ & $<0.01$ & $1.41 \pm 0.85$ & $0.85 \pm 0.64$ & $<0.05$ \\
\hline Bicaval & $1.15 \pm 0.57$ & $0.56 \pm 0.38$ & $<0.01$ & $1.06 \pm 0.60$ & $0.65 \pm 0.29$ & $<0.01$ \\
\hline Short-axis & $0.32 \pm 0.06$ & $0.50 \pm 0.15$ & NS & $0.72 \pm 0.48$ & $0.16 \pm 0.16$ & $<0.05$ \\
\hline \multicolumn{7}{|l|}{ Inferior-posterior rim/defect size } \\
\hline Four chamber & $1.43 \pm 0.94$ & $0.73 \pm 0.65$ & $<0.01$ & $1.28 \pm 0.63$ & $0.75 \pm 0.58$ & $<0.01$ \\
\hline Bicaval & $1.23 \pm 0.55$ & $0.62 \pm 0.44$ & $<0.01$ & $1.17 \pm 0.76$ & $0.66 \pm 0.43$ & $<0.01$ \\
\hline Short-axis & $1.79 \pm 0.52$ & $0.85 \pm 0.97$ & $<0.01$ & $1.19 \pm 1.03$ & $0.87 \pm 0.26$ & $<0.05$ \\
\hline \multicolumn{7}{|l|}{ Defect size/total septal length } \\
\hline Four-chamber & $0.30 \pm 0.11$ & $0.48 \pm 0.16$ & $<0.01$ & $0.29 \pm 0.90$ & $0.44 \pm 0.13$ & $<0.01$ \\
\hline Bicaval & $0.32 \pm 0.09$ & $0.51 \pm 0.15$ & $<0.01$ & $0.33 \pm 0.10$ & $0.50 \pm 0.15$ & $<0.01$ \\
\hline Short-axis & $0.32 \pm 0.06$ & $0.50 \pm 0.15$ & $<0.01$ & $0.40 \pm 0.06$ & $0.42 \pm 0.12$ & NS \\
\hline
\end{tabular}

Data are expressed as mean \pm standard deviation; NS - not significant

\section{Results}

\section{General characteristics}

The average age of a total of 216 patients was $22.5 \pm 15.6$ years (3-67 years) while 124 (57\%) of them were female. The number of pediatric patients was 115 (53\%) with an average age of $10.1 \pm 4.0$ years while the number of adult patients was 101 (47\%) with an average of $36.6 \pm 11.4$ years. The follow-up period was between 1 month and 6 years (median 4.5 years). Sixty-five children and 65 adults underwent closure while it was abandoned in 50 children and 36 adults. Before closure, average ASD diameter measured by balloon sizing and the mean device size were found to be 19.32 $\pm 5.45 \mathrm{~mm}(9-33 \mathrm{~mm})$ and $18 \mathrm{~mm}(8-33 \mathrm{~mm})$, respectively. In $130(60.1 \%)$ of the cases, the defect was closed by the device while 86 cases (39.8\%) were not appropriate for percutaneous defect closure (Figure 1). The FSO device was used in 39 children and 49 adults, and the ASO was used in 26 children and 16 adults. Three of the adult cases had lipo- matous hypertrophy of the interatrial septum. Closure was abandoned during TEE in 39 patients (45.3\%), during balloon sizing in 29 (33.7\%) patients and during the device placement in 18 (21\%) patients. Closure was abandoned due to large defect size in 57 cases, insufficient rim in 8 cases, arrhythmia during the operation in 1 case and inadequate position of the device in the septum in 11 cases. Demographic and clinical features, and closure data of the children and adults are given in Table 1.

\section{Morphology of atrial septal defect and surrounding tissue}

In pediatric cases, measurements in the four-chamber and bicaval section revealed that the superior-anterior rim was significantly longer in cases undergoing closure ( $p<0.05, p<0.05$, respectively). In adult cases, length of the superior-anterior rim was similar in cases undergoing closure and in those not undergoing closure. While the 
length of the inferior-posterior rim was found to be significantly longer in children undergoing closure, the length of the inferior-posterior rim was similar in adults undergoing closure and not undergoing closure.

\section{Echocardiographic ratios}

Both in pediatric and adult cases, the ratio of defect size to total septum length was significantly lower, and the ratio of inferior-posterior rim to the defect was higher in cases undergoing closure. The four-chamber and bicaval measurements revealed that the ratio of superior-anterior rim to the defect was significantly higher in pediatric and adult cases undergoing closure. Measurements of atrial septal defect, total septum length, length of rims surrounding the defect, ratio of atrial septal defect to total septum, ratio of superior-anterior rim to the defect and the ratio of inferior-posterior rim to the defect in different echocardiographic windows in pediatric and adult cases and comparisons between the groups are shown in Table 2.

Both in pediatric and adult cases, the ratio of successful closure was found to be significantly higher when the ratio of defect size to total septum length was $\leq 0.35$, the ratio of superior-anterior rim to the defect size was $>0.75$ and the ratio of inferior-posterior rim to the defect size was $>1.0$ ( $p<0.01)$. Comparisons between closure success and the ratio of defect size to total septum, the ratio of superior-anterior rim to defect size and the ratio of inferior-posterior rim to defect size are shown for all the cases in Table 3.

\section{Predictive factors}

An analysis by classification into either group by multivariate logistic regression analysis and by contingency tables identified ASD/TS ratio $\leq 0.35$, and ratio of superior-anterior rim to ASD > 0.75 and ratio of inferior-posterior rim to $A S D>1.0$ as factors predictive of successful ASD occlusion, as shown in Table 3. When a predictive factor analysis test was performed after categorization, the ratio of closure success was $64.6 \%$ in cases having one or more of these predictive parameters. The ratio of closure success was $71.6 \%$ in cases having two or more of these predictor parameters and $100 \%$ in cases where all these predictive parameters were confirmed. No successful closure was performed in cases having none of these predictive parameters. It was found that having more than one of these predictors in a single case increased the chance of closure success significantly $(p<0.001)$.

\section{Morphologic characteristics of defect and atrial septal rims}

When all the cases included in the study were evaluated, it was found that the defect was centrally located in 67 (31\%) cases while the most frequently encountered rim deficiency was superior-anterior rim deficiency (26.3\%). When those not undergoing closure were evaluated, it was found that the defect was centrally located in 3 (3.4\%) cases and
Table 3. Comparisons between closure success and ratio of superior-anterior rim to ASD size, ratio of inferior-posterior rim to ASD size, and ratio of ASD size to total septal length

\begin{tabular}{|c|c|c|c|}
\hline Parameter & $\begin{array}{c}\text { Successful } \\
\text { closure } \\
n(\%)\end{array}$ & $\begin{array}{c}\text { Failed } \\
n(\%)\end{array}$ & $\begin{array}{c}\text { Value } \\
\text { of } p\end{array}$ \\
\hline \multicolumn{4}{|c|}{ Anterior-superior rim/defect size } \\
\hline$\leq 0.5$ & 35 (26.9) & $49(56.9)$ & $<0.05$ \\
\hline$>0.5$ & $95(73.1)$ & $37(43.0)$ & $<0.05$ \\
\hline$\leq 0.75$ & $53(40.7)$ & $64(74.4)$ & $<0.05$ \\
\hline$>0.75$ & $77(59.2)$ & $22(25.6)$ & $<0.01$ \\
\hline$\leq 1.0$ & $78(60.0)$ & $75(87.2)$ & $<0.05$ \\
\hline$>1.0$ & $82(63.0)$ & $11(12.8)$ & $<0.01$ \\
\hline \multicolumn{4}{|c|}{ Posterior-inferior rim/defect size } \\
\hline$\leq 1.0$ & $79(60.8)$ & $77(89.5)$ & NS \\
\hline$>1.0$ & $51(39.2)$ & $9(10.5)$ & $<0.01$ \\
\hline$\leq 1.4$ & $97(74.6)$ & $82(95.3)$ & NS \\
\hline$>1.4$ & $33(25.4)$ & $4(4.7)$ & $<0.05$ \\
\hline \multicolumn{4}{|c|}{ Defect size/total septal length } \\
\hline$\leq 0.3$ & $\leq 0.3$ & $\leq 0.3$ & $\leq 0.3$ \\
\hline$>0.3$ & $>0.3$ & $>0.3$ & $>0.3$ \\
\hline$\leq 0.35$ & $\leq 0.35$ & $\leq 0.35$ & $\leq 0.35$ \\
\hline$>0.35$ & $>0.35$ & $>0.35$ & $>0.35$ \\
\hline$\leq 0.4$ & $\leq 0.4$ & $\leq 0.4$ & $\leq 0.4$ \\
\hline$>0.4$ & $>0.4$ & $>0.4$ & $>0.4$ \\
\hline \multicolumn{4}{|c|}{ Count of predictive factors ${ }^{*}$} \\
\hline$\geq 3$ & $78(60)$ & $0(0)$ & $<0.001$ \\
\hline$\geq 2$ & $121(93.0)$ & $48(55.8)$ & $<0.001$ \\
\hline$\geq 1$ & $130(100)$ & $71(82.5)$ & $<0.05$ \\
\hline 0 & $0(0)$ & $15(17.4)$ & $<0.001$ \\
\hline
\end{tabular}

Data are expressed as frequencies (percentile); NS - not significant. *Ratio of superior-anterior rim to defect size $>0.75$, ratio of inferior-posterior rim to defect size $>1.0$, and defect size/total septal length ratio $\leq 0.35$

the most frequently encountered rim deficiency was inferior-posterior rim deficiency (33.7\%). There was no rim deficiency in $83(63.8 \%)$ of the cases undergoing closure while $47(36.1 \%)$ of the said cases had anterior-superior rim deficiency. The majority of cases having superior-anterior rim deficiency had a successful closure. It was found that closure was abandoned in case of having an insufficiency in at least one rim other than the superior-anterior rim. Types and numbers of defect morphologies are shown for all cases in Table 4.

\section{Discussion}

In the present study, it was found that the ratio of the defect to total septum length and the ratio of superior-anterior and inferior-posterior rims to the defect are echocardiographic predictors that can be used in transcatheter ASD closure both in pediatric and adult patients, and that 
Table 4. Types and numbers of defect morphologies

\begin{tabular}{|c|c|c|}
\hline Variable & $\begin{array}{l}\text { Unsuccessful closure } \\
\qquad(N=86), n(\%)\end{array}$ & $\begin{array}{l}\text { All patients } \\
(N=216), n(\%)\end{array}$ \\
\hline \multicolumn{3}{|l|}{ Morphological characteristics of atrial septum } \\
\hline Centrally placed defects & $3(3.4)$ & $67(31)$ \\
\hline Deficient superior-anterior rim & $10(11.6)$ & $57(26.3)$ \\
\hline Deficient inferior-posterior rim & $29(33.7)$ & $29(13.4)$ \\
\hline Perforated aneurysmatic septum & $5(5.8)$ & $15(7.0)$ \\
\hline Multiple defects & $5(5.8)$ & $16(7.4)$ \\
\hline Deficient inferior-anterior and superior-anterior rim & $8(9.3)$ & $9(4.2)$ \\
\hline Deficient inferior-posterior and posterior rim & $11(12.7)$ & $11(5.1)$ \\
\hline Deficient inferior-anterior rim & $8(9.3)$ & $8(3.7)$ \\
\hline Deficient superior-posterior rim & $6(7.0)$ & $6(2.7)$ \\
\hline Deficient coronary sinus rim & $1(1.1)$ & $1(0.4)$ \\
\hline \multicolumn{3}{|l|}{ Count of deficient rim } \\
\hline Single rim deficiency (superior-anterior rim) & $10(11.6)$ & $57(26.3)$ \\
\hline Single rim deficiency (other than superior-anterior rim) & $20(23.2)$ & $20(9.2)$ \\
\hline Two deficient rims (superior-anterior rim + other rims) & $31(36.0)$ & $31(14.3)$ \\
\hline Two deficient rims (none is superior-anterior rim) & $10(11.6)$ & $10(4.6)$ \\
\hline Three deficient rims (superior-anterior rim + two other rims) & $5(5.8)$ & $5(2.3)$ \\
\hline Three deficient rims (none is superior-anterior rim) & $3(3.4)$ & $3(1.4)$ \\
\hline Four deficient rims (superior-anterior rim + three other rims) & $4(4.6)$ & $4(1.8)$ \\
\hline Four deficient rims (none is superior-anterior rim) & $0(0)$ & $0(0)$ \\
\hline
\end{tabular}

Data are expressed as frequencies (percentile)

the larger the number of predictor factors, the greater the chance for successful closure.

Atrial septal defects are treated either by transcatheter or surgical closure. As the rims surrounding the defect have a solid tissue structure, the waist of the device stents the defect and the device is positioned in the septum properly. While some of the characteristics of the defect and surrounding septal tissue can be obtained by echocardiography, one has to use TEE to obtain all the characteristics [4]. Acar et al. reported that maximal diameter of the defect and the dimensions of the septal rims were essential parameters for the selection of optimal cases for closure [7]. In a meta-analysis study published by Kleinman, it was emphasized that TEE was the gold standard in transcatheter closure in ASD and that it should be used in evaluating the defect and rims during the process [5]. Rosenfeld et al. reported that the only factor having an effect on percutaneous ASD closure was the defect size and that all the cases having a defect size of $\leq 13 \mathrm{~mm}$ had effective closure while the success rate was $59 \%$ in cases having defect diameter $>13 \mathrm{~mm}$ [8]. The reason for reporting $13 \mathrm{~mm}$ as the threshold for the defect size in the said study may be related to not having advanced and practical closure devices in those days and acting conservatively as the long-term results were not known.
Naming a definite ASD size or predictor would not be logical as the ASD size, localization and structure of rims differ among patients. Therefore, determining other predictors along with measuring ASD size and evaluating the closure using these predictors would increase the rate of success. In this respect, Reddy et al. reported that final evaluation in percutaneous closure should be made by measuring the defect size through balloon sizing and that the ratio of ASD/TS and superior-anterior rim/ASD were higher in the closure group [9]. In our study, similar to the study of Reddy et al., TEE revealed that both the average defect size and the stretched defect size in balloon sizing were significantly lower in the closure group while the total septal lengths of the groups were similar.

In a study on the morphology of defects, Podnar et al. emphasized that the most frequent rim deficiency was superior-anterior rim deficiency (42.1\%) and that the defect size was the most effective predictor for a successful closure [6]. Sahebjam et al. observed that superior-anterior rim deficiency, which was present in 48 (24\%) cases, was the most frequent morphological variation [10]. Device closure was performed successfully in 32 (66.6\%) of their patients. In our study, morphological evaluation of ASDs by TEE revealed that the defect was centrally localized in 67 (31\%) cases while the most frequent (25\%) rim deficiency was superior-an- 
terior rim deficiency both in pediatric and adult cases. A successful closure was performed in the majority of cases $(82.4 \%)$ having superior-anterior rim deficiency. Thus, it was observed that having a superior-anterior rim deficiency did not affect transcatheter closure of ASD significantly. In 3 of the adult cases having lipomatous hypertrophy, the device was observed to be in an appropriate position holding on to the lipomatous hypertrophic rims after it was released and the defect was closed successfully once the device came back to its original configuration. In patients having lipomatous hypertrophy of the interatrial septum, closure device returning to its original configuration (shape memory) due to an elastic structure and having a selfcentering mechanism that keeps the device centered in the defect make closure of such defects possible [11].

The strength of the rims surrounding the defect is important both for the device to stent the defect properly and to hold the septal rims adequately. Therefore, the ratio of superior-anterior rim/ASD and inferior-posterior rim/ASD can be used as a relative indicator of the strength of the soft tissue surrounding the defect. In our study, no successful ASD closure could be performed in cases where the ratio of defect to total septum was more than 0.35 while a successful closure was performed in cases having a ratio lower than 0.35 . Similarly, Reddy et al. reported that closure was not performed in cases having a high ASD/TS ratio and found that the length of the inferior-posterior rim was similar in both groups [9]. In our study, the ASD/TS ratio was found to be low both in pediatric and adult cases undergoing percutaneous closure while the superior-anterior rim/ASD ratio and inferior-posterior rim/ASD ratio were significantly high. A successful closure was performed in cases where the length of the superior-anterior rim was longer than the ASD (ratio greater than 1). Multivariate logistic regression analysis performed to determine whether the three predictors, namely the ASD/TS ratio, the superior-anterior $\mathrm{rim} / \mathrm{ASD}$ ratio and the inferior-posterior rim/ASD ratio, could be used as predictors in transcatheter defect closure revealed there was a strong predictive association between successful closure and the said predictors. In contrast to our findings, Reddy et al. found that the inferior-posterior rim/ ASD ratio was not a reliable predictor [9]. This could be related to having a limited number of cases and having no advanced devices for a successful closure on those days.

In this study, ASD/TS $\leq 0.35$, superior-anterior rim/ASD ratio $>0.75$ and inferior-posterior rim/ASD ratio $>1$ were found to be reliable predictive values both in children and adults. While the ratio of successful closure was $64.6 \%$ in cases having one or more of the predictive parameters, the said ratio was $71.6 \%$ in those having two or more of the said predictors. The success rate was $100 \%$ in cases where all the said parameters were confirmed. There was no single case having none of these predictive parameters but a successful closure. Having more than one of these predictors increased the success ratio of the transcatheter closure, which confirms that these are reliable and practical parameters to be used as predictive parameters for a successful transcatheter closure.

In our study, closure was performed in 65 and abandoned in 50 of the pediatric cases. In adult cases, 65 of the patients underwent closure while it was abandoned in 36 patients. The reason for having a low closure rate is the detailed evaluation performed by TEE not only in those who were likely to undergo closure but all the ASD cases.

\section{Conclusions}

In the present study, a ratio of the defect size to total septum length $\leq 0.35$, a ratio of superior-anterior rim to defect size $>0.75$ and a ratio of inferior-posterior rim to defect size $>1.0$ were found to be echocardiographic predictors that could be used in successful transcatheter ASD closure both in children and adults. It was concluded that having more predictors increased the chance of a successful closure.

\section{References}

1. Fyler DC. Atrial septal defect secundum. In: Pediatric cardiology. Nadas, Fyler DC (eds.). W.B. Sounders, Philadelphia 1972; 513-524.

2. Porter CJ, Edwards WD. Atrial septal defects. In: Moss and Adam's heart disease in infants, children and adolescent: including the fetus and young adults. Allen HD, Shaddy R, Driscoll D, Feltes T (eds.). Lippincott Williams and Wilkins, Philadelphia 2008; 632-644.

3. Prokselj K, Kozelj M, Zadnik V. Echocardiographic characteristics of secundum-type atrial septal defects in adult patients: implications for percutaneous closure using amplatzer septal occluders. J Am Soc Echocardiogr 2004; 17: 1167-1172.

4. Kadron RE, Sokoloski MC, Levi D, et al. Transthoracic echocardiographic guidance of transcatheter atrial septal defect closure. Am J Cardiol 2004; 94: 256-260.

5. Kleinman CS. Echocardiographic guidance of catheter-based treatments of atrial septal defect: transesophageal echocardiography remains the gold standard. Pediatr Cardiol 2005; 26: 128134.

6. Podnar T, Martanovic P, Gavora P, et al. Morphological variations of secundum-type atrial septal defects: feasibility for percutaneous closure using Amplatzer septal occluders. Cathet Cardiovasc Intervent 2001; 53: 386-391.

7. Acar P, Saliba Z, Bonhoeffer B, et al. Influence of atrial septal defect anatomy in patient selection and assessment of closure with the Cardioseal device: a three-dimensional transesophageal echocardiographic reconstruction. Eur Heart J 2000; 21: 573-581.

8. Rosenfeld HM, van der Velde ME, Sanders SP, et al. Echocardiographic predictors of candidacy for successful transcatheter atrial septal defect closure. Cathet Cardiovasc Diagn 1995; 34: 29-34.

9. Reddy SCB, Rao PS, Ewenko J, et al. Echocardiographic predictors of success of catheter closure of atrial septal defect with the buttoned device. Am Heart J 1995; 129: 76-82.

10. Sahebjam M, Zavar R, Lotfi-Tokaldany M, et al. Transesophageal echocardiographic characteristics of secundum-type atrial-septal defect in adult patients. J Teh Univ Heart Ctr 2009; 4: 240-243.

11. Moir WS, McGaw DJ, Harper RW, et al. Atrial septal defect device closure in a patient with lipomatous hypertrophy of the atrial septum. Circulation 2003; 107: e217. 